Regards sur l'économie allemande

Bulletin économique du CIRAC

$71 \mid 2005$

Varia

\title{
Eglises : en quête d'une nouvelle compétitivité
}

\section{Isabelle Bourgeois}

\section{OpenEdition}

Journals

Édition électronique

URL : http://journals.openedition.org/rea/346

DOI : $10.4000 /$ rea.346

ISBN : 978-2-8218-0838-6

ISSN : 1965-0787

Éditeur

CIRAC

Édition imprimée

Date de publication : 1 mai 2005

Pagination : 32-34

ISSN : 1156-8992

Référence électronique

Isabelle Bourgeois, "Eglises : en quête d'une nouvelle compétitivité », Regards sur l'économie allemande [En ligne], 71 | mai 2005, document 3, mis en ligne le 22 avril 2008, consulté le 15 septembre 2020. URL : http://journals.openedition.org/rea/346

Ce document a été généré automatiquement le 15 septembre 2020.

(C) CIRAC 


\title{
Eglises : en quête d'une nouvelle compétitivité
}

\author{
Isabelle Bourgeois
}

\section{Des acteurs majeurs dans le champ social et économique}

1 Contrairement à ce que laisse entendre son titre, il est consacré aux Eglises protestante et catholique d'Allemagne, dont les 'parts de marché' sont à peu près équivalentes outre-Rhin, bien qu'inégalement réparties sur le territoire. Dès lors qu'on fait abstraction de leur mission spirituelle pour ne retenir que les activités séculières de ces deux organisations concurrentes qui œuvrent toutes deux dans le domaine qu'il est convenu d'appeler les "services d'intérêt général", le rapprochement sous une dénomination unique («Eglise») semble justifié. Aussi bien l'Eglise catholique que l'Eglise protestante d'Allemagne (Evangelische Kirche in Deutschland, EKD) sont en effet des acteurs majeurs dans le champ social et économique outre-Rhin: leurs organisations caritatives Diakonie (protestante) et Caritas (catholique) occupent 450000 et 495000 salariés respectivement à elles seules. Réunies, les Eglises constituent le premier prestataire de services d'Allemagne, ce qui amène naturellement à les étudier sous l'angle économique et à les considérer aussi comme des entreprises. L'ouvrage se contente de développer une approche de plus en plus répandue, non seulement en raison de la 'puissance' économique et sociale que représentent les Eglises outre-Rhin, mais aussi parce qu'elles se sont lancées depuis peu dans ce qu'il faut bien appeler une 'quête de compétitivité'. L'Eglise protestante, suivie de l'Eglise catholique, n'ont pas hésité à faire appel aux compétences du cabinet Mc Kinsey qui, selon diverses sources, offrirait ses compétences à titre gracieux ... 


\section{Dans le système de protection sociale allemand, ...}

2 Les Eglises allemandes, financées par un impôt prélevé pour leur compte par les services fiscaux (Kirchensteuer) en vertu du régime du concordat, sont présentes dans de nombreuses activités relevant de la culture, du domaine social, dont l'accueil de la petite enfance (jardins d'enfants), la formation scolaire et universitaire, sans parler de leur rôle dans le système hospitalier ou les infrastructures d'accueil des personnes âgées ou encore dans la lutte contre l'exclusion. Elles ont ainsi un rôle central dans l'organisation des structures de la protection sociale, aux côtés des pouvoirs publics (Bund, Länder, communes), de l'Arbeiterwohlfahrt - l'action sociale ouvrière proche du mouvement ouvrier (145000 salariés) - et d'institutions privées à but non lucratif comme les fondations (par exemple la Fondation Krupp dans le domaine hospitalier).

\section{... elles assument des missions d'assistance}

3 Plus fondamentalement encore, elles sont l'un des deux piliers sur lequel repose l'Etat providence allemand, ainsi que le rappelle par exemple le syndicat IG Metall dans l'argumentaire soumis à la réflexion des participants à son "Congrès sur l'Etat social " des 6 et 7 avril 2005. Le premier pilier est constitué par les systèmes de protection sociale hérités des lois sociales adoptées sous Bismarck, et "réservés aux salariés ainsi qu'à leur famille ». Au principe d'assurance de ce pilier s'en ajoute un autre, "né de la tradition communale et ecclésiastique de l'assistance aux pauvres", et qui représente le second pilier, avec pour acteurs principaux les communes et les Eglises.

\section{Des corps intermédiaires importants dans le jeu démocratique}

Les Eglises (de même que la communauté juive) ont également, puisqu'elles font partie des "groupes représentatifs de la société » (gesellschaftlich relevante Gruppen) aux côtés des syndicats, des fédérations professionnelles et autres (parents d'élèves, par exemple), un rôle sociétal important en qualité de corps intermédiaires. C'est ainsi qu'elles sont très présentes dans le jeu démocratique, via leur participation institutionnelle à la régulation de l'audiovisuel, leurs activités d'organisateurs et d'animateurs du débat sociétal (via par exemple les "académies" de Loccum pour l'Eglise protestante ou de Trèves pour l'Eglise catholique, pour n'en citer que deux), leurs émissions télévisées, ou en tant que propriétaires de maisons d'édition et surtout d'agences d'information: l'Evangelischer Pressedienst et la Katholische Nachrichtenagentur.

\section{Elles sont au cœur de la réforme de l'Etat providence}

Elles se trouvent donc aujourd'hui doublement au cœur d'une réforme du système de l'Etat providence allemand: en tant qu'acteurs non étatiques, elles sont amenées à relayer plus que par le passé des régimes publics en mal de financement; en tant qu'acteurs représentatifs de la société, impliqués en amont dans le processus de prise de décision tout comme les syndicats, elles ont à co-définir les orientations de la 
politique sociale allemande. Mais normalement, elles agissent en tant qu'institutions indépendantes, diffusant dans l'espace public des textes soumis à réflexion collective, ou via leurs réseaux de relais dans l'opinion comme auprès des décideurs.

\section{Et l'EKD est associée aux réflexions d'IG Metall}

6 Il est inhabituel qu'un de leurs représentants soit associé ès qualités au processus de réflexion d'un autre corps intermédiaire, surtout d'un syndicat. Ce fut pourtant le cas de Wolfgang Huber, président du conseil de l'EKD, qui participa aux travaux du «Congrès sur l'Etat social» d'IG Metall. En octobre dernier encore, W. Huber (et son homologue catholique, le cardinal Lehmann, lui aussi convié) avait refusé l'invitation, arguant du fait que le mouvement syndical se préoccupait trop des intérêts des seuls salariés, et l'invitant «à prendre ses responsabilités au nom de l'intérêt de l'ensemble de la collectivité». Le quotidien Frankfurter Allgemeine Zeitung (27-10-04)s'était empressé de rapporter ces propos dans le contexte du débat sur un système de protection sociale trop exclusivement assis sur le financement par les salaires et les reproches faits aux syndicats de privilégier les insiders au détriment des outsiders. Mais entre temps, le syndicat IG Metall avait changé d'approche (voir REA 69/05) pour investir désormais le champ social.

\section{Des revenus fiscaux et d'importantes ressources propres}

7 Les revenus fiscaux des Eglises catholique et protestante s'élevaient en 2004 à respectivement 4,0 milliards $€$ et 3,7 milliards $€$ (estimations, Schwarz, 2005). Ils couvrent les dépenses liées à leurs missions spirituelles et financent une partie de leurs activités dans le domaine de la formation, ainsi que le rappelle un dossier publié par le quotidien économique Handelsblatt (1/3-10-2004). Certes, le volume total des recettes fiscales a baissé de 1 milliard $€$ depuis 1995, la diminution affectant surtout l'Eglise protestante, qui a vu ces ressources baisser de 600 millions $€$ durant la même période. Cette évolution reflète la baisse du nombre de foyers fiscaux redevables de cet impôt (les contribuables déclarent leur appartenance à l'une ou l'autre des Eglises), surtout dans les nouveaux Länder; il s'agit principalement de chômeurs cherchant à réaliser des économies et qui donc optent contre l'appartenance à une Eglise). Elle est cependant moins dramatique pour le bilan des Eglises que ne le laissent entendre la plupart des commentaires. Car elles disposent de trois autres sources de financement : les dons et donations des particuliers et entreprises, diverses aides d'Etat (subventions et exonérations), et leurs ressources propres, pour l'essentiel issues d'activités économiques.

\section{Rétribution des services et revenus immobiliers}

Selon le bilan 2004 de l'EKD, détaillé dans l'ouvrage de Schwarz, la part de l'impôt d'église dans ses recettes (total : 10,5 milliards $€$ ) ne s'élevait qu'à $43,7 \%$. Celle des dons et donations frisait $20 \%$, de même que la part des «rétributions pour services ecclésiastiques » (jardins d'enfants, écoles, accueil des personnes âgées notamment) et 
des revenus immobiliers. Si, pour des raisons structurelles, la comparaison avec l'Eglise catholique n'est pas possible, on connaît néanmoins le budget de divers achevêchés. Celui de Cologne par exemple, notoirement le plus riche d'Allemagne, s'élevait en 2003 à 679 millions $€$, dont 462 millions $€$ de recettes fiscales. Quant à la valeur de son patrimoine, comment la chiffrer? Un expert s'est risqué à l'exercice, estimant la valeur vénale (!) de la cathédrale de Cologne à 500 millions $€ . .$.

\section{Un marché à fort potentiel : la dépendance liée à l'âge}

Parmi les activités de services 'sociaux', le secteur de la dépendance est non seulement important, mais amené à se développer considérablement sous le double effet du vieillissement démographique et de la crise du financement des systèmes de protection sociale. D'ores et déjà, $65 \%$ du 'marché' des infrastructures hospitalières comme des maisons de retraite sont gérés par le dispositif caritatif des Eglises et de l' Arbeiterwohlfahrt. Le seul groupe Augustinum (Diakonie), leader (protestant) dans le segment des maisons de retraite, y a une part de marché de $20 \%$, à en croire son président, Markus Rückert (Süddeutsche Zeitung, 15-11-04). Le groupe, qui réalise un CA de 260 millions $€$, gère 21 maisons de retraite accueillant 7000 pensionnaires. Markus Rückert compte fermement sur la réforme à venir de l'assurance dépendance (voir REA 68/04) pour développer ses parts sur un 'marché' à forte perspective de croissance. Mais il regrette que ce marché ait été sur-régulé, allant jusqu'à estimer que le segment les institutions d'accueil du grand âge est "soumis aux règles de l'économie planifiée » alors que son groupe, à l'inverse, "obéit aux lois de la concurrence, cherchant à conquérir les clients un à un ». Ce discours n'a rien d'étonnant: M. Rückert est membre du directoire de la Fédération du patronat allemand (BDA). Mais son groupe, talonné par le français Marseille-Kliniken AG (CA d'environ 200 millions $€$ ) et une multitude de plus petits prestataires (dont ceux de l'Eglise catholique, via Caritas), devra affronter prochainement la concurrence américaine qui se prépare à prendre pied sur ce marché à fort potentiel.

\section{Les réformes sociales touchent aujourd'hui les Eglises}

10 Pour un ensemble de raisons - dont le vieillissement démographique, le déficit structurel des systèmes de protection sociale, le recours accru à la prévoyance individuelle, la libéralisation en cours du marché des services d'intérêt (économique) général- les Eglises allemandes ont commencé à découvrir les méthodes de management. Elles cherchent aujourd'hui à mieux structurer leurs activités, séparant plus nettement les activités marchandes et non marchandes et visent à réaliser des gains de productivité. En réduisant leurs coûts, comme toute entreprise (Handelsblatt, 1/3-10-04). L'archevêché de Cologne a décidé de réaliser 90 millions $€$ d'économies par an à partir de 2006, celui d'Aix-la-Chapelle, de réduire ses effectifs de 200 salariés... Si les Eglises allemandes, qui sont des collectivités de droit public dotées d'un statut particulier qui les soustrait au jeu de la négociation conventionnelle de branche, gèrent les plans sociaux en interne, elles ne s'en heurtent pas moins à l'obstacle commun à tout le secteur caritatif. Rêvant ouvertement d'une culture de négociation à la Siemens, le théologien et économiste Markus Rückert décrit l'obstacle ainsi : une approche de la 
GRH «en termes de statuts de cadres moyens de la fonction publique ». Désormais, les réformes sociales allemandes touchent aussi les Eglises.

\section{RÉSUMÉS}

« Avec un patrimoine supérieur à 500 milliards $€, 53$ millions de membres et plus de 1,3 million de salariés, répartis sur près de 50000 entreprises indépendantes, et un chiffre d'affaires annuel dépassant les 125 milliards $€$, l'Eglise est un empire économique qui se place en tête du palmarès des plus grands groupes allemands ». Cette citation, tirée de l'édition dominicale Welt am Sonntag (29-12-2003) du quotidien du même nom, est au cœur d'un ouvrage qui vient de paraître aux éditions Campus, spécialisées dans l'économie et le management (Friedhelm Schwarz, Wirtschaftsimperium Kirche. Der mächtigste Konzern Deutschlands).

\section{INDEX}

Mots-clés : compétitivité, église, religion, système social 